\title{
Low-frequency quantum oscillations from interactions in layered metals
}

\author{
Andrew A. Allocca $\circledast^{1, *}$ and Nigel R. Cooper ${ }^{1,2}$ \\ ${ }^{1}$ TCM Group, Cavendish Laboratory, University of Cambridge, J. J. Thomson Avenue, Cambridge CB3 OHE, United Kingdom \\ ${ }^{2}$ Department of Physics and Astronomy, University of Florence, Via G. Sansone 1, 50019 Sesto Fiorentino, Italy
}

(Received 22 March 2021; revised 4 August 2021; accepted 23 September 2021; published 28 October 2021)

\begin{abstract}
Metals composed of weakly coupled, stacked layers possess a Fermi surface that slightly varies in size along the stacking direction. This appears in de Haas-van Alphen (dHvA) oscillations of the magnetization with a magnetic field as two close frequencies, corresponding to the two extremal Fermi-surface cross-sectional areas. We show that, for layered materials of sufficiently high mobility, Coulomb interactions can have a dramatic effect on the form of the dHvA oscillations: There is also generically an oscillation at the small difference of the two large frequencies. We determine the size and form of this effect, and show that it probes the short-range part of the Coulomb interactions within the layered material. We argue that this interaction effect may explain recent experimental observations of anomalous low-frequency $\mathrm{dHvA}$ oscillations in the ultrapure delafossites.
\end{abstract}

DOI: 10.1103/PhysRevResearch.3.L042009

Introduction. Modern materials science has produced a wide variety of new sorts of solid state systems, such as those characterized by nontrivial topology or strong interactions. The novel properties of these materials have led to reexaminations of previously well-understood phenomena. Quantum oscillations (QOs) of the magnetization as a function of (inverse) magnetic field [1], long interpreted in terms of the geometry of closed Fermi surfaces, and the standard LifshitzKosevich (LK) theory [2], have been necessarily reexplored following the discovery of Weyl and nodal semimetals [3-8], quasiperiodic systems $[9,10]$, and the observation of QOs in insulators [11-18], systems all featuring unusual or absent Fermi surfaces.

Following in this vein, recent de Haas-van Alphen (dHvA) studies on delafossites, a class of layered materials featuring strong interactions and high in-plane mobility, also display anomalous behavior-large, low-frequency QOs at the difference of the two natural high frequencies related to extremal Fermi-surface areas $[19,20]$. These observations are not readily explained by existing theory based on magnetic interactions [21], and require a reexamination of the theory of the dHvA effect in these new materials settings.

In this Letter, we show that Coulomb interactions can lead to difference-frequency oscillations of the magnetization, of a size that can account for these experimental observations. The mechanism that we identify is often disregarded in treatments of interaction effects on the dHvA effect [21-23], as it requires the retention of terms that are smaller than those needed to account for the high-frequency oscillations. How-

\section{*aa2182@cam.ac.uk}

Published by the American Physical Society under the terms of the Creative Commons Attribution 4.0 International license. Further distribution of this work must maintain attribution to the author(s) and the published article's title, journal citation, and DOI. ever, in high-mobility systems such as the delafossites, the dominant dHvA oscillation at the difference frequency in both bilayer and multilayer systems can arise from the Coulomb interaction mechanism we identify. In these circumstances our theory shows that the measurement of a difference-frequency oscillation is a signature of strong nonlocal interactions. Our results indicate that, in general, additional considerations are warranted when analyzing dHvA oscillations in high-mobility metals.

To trace the origin of a difference frequency in QOs, first consider a noninteracting Fermi gas whose Fermi surface has two extremal orbits of similar areas. As the magnetic field varies, the Landau quantized orbits sweep through these two extremal areas, causing thermodynamic quantities to oscillate at the two frequencies $f_{ \pm}$. Within a model of noninteracting electrons at fixed chemical potential these oscillations are independent. However, any nonlinearities that couple these two oscillations at $f_{ \pm}$, can lead to oscillations at the difference frequency $\delta f=\left|f_{+}-f_{-}\right|$(as well as sidebands). One source of nonlinearity is the magnetic interaction, by which the magnetic field $B=\mu_{0}(H+M)$ acquires an oscillatory component through the oscillating magnetization $M(H)$. This leads to a difference-frequency component of size related to the geometry-dependent demagnetization field [21,24]. However, this effect is insufficient to explain the difference-frequency oscillations of magnetization seen in recent experiments $[19,20]$.

As we will show, a much larger difference-frequency oscillation can arise from Coulomb interactions. One consequence of the Coulomb interaction is that its long-range component forces overall charge neutrality in bulk threedimensional (3D) materials, requiring one to work at a fixed electron number density $n$ rather than a fixed chemical potential. This introduces a nonlinearity, mediated by oscillations in the chemical potential $[25,26]$, that can readily produce a difference-frequency oscillation in the conductivity (Shubnikov-de Haas effect) [27], or in the magnetization 
(dHvA effect) in multiband systems [28-32]. We find, however, that in our system of interest the difference-frequency oscillation of the magnetization from this effect alone is rather small. In fact, as we will show, the dominant effect arises from a full consideration of the Coulomb interactions that also takes account of the (screened) short-range interactions coupling local fluctuations of the charge density. The combination of both short- and long-range components of the Coulomb interaction produces a much larger difference-frequency oscillation than either does independently. We emphasize that both interaction effects are required to produce a sizable dHvA effect, which we consider here; for the Shubnikov-de Haas effect a large difference frequency oscillation can be readily obtained by fixing density alone, without invoking a short range Coulomb interaction [27].

Bilayer (2D). To illustrate the essential physics underlying this mechanism, we first consider a bilayer model. (We neglect possible strongly correlated phases that can arise from strong interactions in 2D Landau quantized systems.) We consider a pair of identical, parallel 2D gases of spinless electrons, with a parabolic in-plane dispersion of effective mass $m^{*}$. Interlayer hopping, of amplitude $\Delta / 2$, splits the energy eigenstates into symmetric and antisymmetric subbands, and a perpendicular magnetic field $B$ reorganizes the in-plane states into Landau levels. The spectrum is then $\epsilon_{l, s}=\hbar \omega_{c}(l+1 / 2)-s \Delta / 2$, with $\omega_{c}=e B / m^{*}$ the cyclotron frequency, $l=0,1,2, \ldots$ indexing Landau levels, and $s= \pm$ marking even and odd subbands. For a chemical potential $\mu$, the densities of the two subbands at $B=0$ are $n_{ \pm}(0)=(\mu \pm \Delta / 2) m^{*} /\left(2 \pi \hbar^{2}\right)$ and there are two distinct Fermi surfaces. At nonzero $B$ these give rise to QOs in the magnetization at frequencies $f_{ \pm}=$ $e n_{ \pm}(0) / h=m^{*}(\mu \pm \Delta / 2) / e \hbar$. Within LK theory these are the only two frequencies in the magnetization oscillations.

At a fixed chemical potential $\mu$, the occupations of the two subbands, $n_{ \pm}(B)$, both oscillate with $B$. For $\hbar \omega_{c} \ll \Delta \ll \mu$, so that many Landau levels are occupied and $n_{ \pm}$have similar average values, these oscillations are on the scale of $n_{\Phi}=$ $B / \Phi_{0}$, the degeneracy of each Landau level per unit area, with $\Phi_{0}=h / e$ the magnetic flux quantum. Keeping only the first harmonic, we write

$$
n_{ \pm}(B) \sim n_{ \pm}(0)-\eta n_{\Phi} \sin \left(2 \pi \frac{\mu \pm \Delta / 2}{\hbar \omega_{c}}\right),
$$

where $\eta \lesssim 1$ is introduced phenomenologically to account for any disorder and temperature effects that suppress oscillations. The oscillations in density Eq. (1) lead to oscillations in the Coulomb interactions that can also have components at the difference frequency. Taking the ionic background charge density to be $n_{I}$ in each well, and computing the expectation value of the electron-electron interactions in the noninteracting ground state leads to the interaction energy

$$
E_{\text {int }} \sim V\left(\bar{n}(B)-n_{I}\right)^{2}-V(\delta n(B) / 2)^{2},
$$

with $\bar{n} \equiv\left(n_{+}+n_{-}\right) / 2$ and $\delta n \equiv n_{+}-n_{-}$. The first term in (2) is the Hartree energy, related to the overall electrostatic energy of fluctuations of the total charge density. The second term is the Fock term, describing the suppression of short-range repulsion due to exchange [33]. Since the subband densities $n_{ \pm}$ oscillate at the frequencies $f_{ \pm}$, the cross term $n_{+} n_{-}$produces a small component that oscillates at the difference frequency $\delta f=\left|f_{+}-f_{-}\right|$, with an amplitude of order $V \eta^{2} n_{\Phi}^{2}$.

To account for the effects of the long-range part of the Coulomb interaction, we enforce charge neutrality, requiring $\bar{n}(B) \equiv n_{I}$. The chemical potential $\mu$ becomes an oscillating function of $B$, which to first order in $\eta$ is

$$
\mu(B) \approx \mu(0)+\frac{\eta \hbar \omega_{c}}{2} \sum_{s= \pm} \sin \left(2 \pi \frac{\mu(0)+s \Delta / 2}{\hbar \omega_{c}}\right) .
$$

With this oscillating $\mu(B)$ inserted in Eq. (1), one finds that the density $n_{ \pm}(B)$ oscillates not just at $f_{ \pm}$but also at the difference frequency $\delta f$ (and at other sidebands). The restriction to fixed $n$ has a large effect on the interaction energy. The Hartree term vanishes exactly, leaving just the Fock exchange energy $-V[\delta n / 2]^{2}$, which contains an oscillatory term of size

$$
\sim V \eta n_{\Phi} \delta n(0) \sum_{s= \pm} s \sin \left(2 \pi \frac{\mu(B)+s \Delta / 2}{\hbar \omega_{c}}\right)+\cdots .
$$

This gives rise to oscillations at the difference frequency $\delta f$, with amplitude of order $V \eta^{2} n_{\Phi} \delta n(0)$. This is larger than the $\delta f$ oscillation we identified at fixed chemical potential by a factor of $\delta n(0) / n_{\Phi}=\Delta /\left(\hbar \omega_{c}\right)$, which can be large at low fields.

Multilayer (3D). We now turn to a model for a multilayer metal. As above, each layer is described by a parabolic dispersion with effective mass $m^{*}$, electrons may hop between adjacent layers with an amplitude that we now denote $t_{\perp}$, and a perpendicular field $B$ breaks in-plane states into Landau levels. At nonzero temperature $T$ the system is described by the action

$$
S_{0}=\sum_{\epsilon_{n}, l, k_{y}} \int_{k_{z}} \bar{\psi}_{l, k_{y}}\left(k_{z}, \epsilon_{n}\right)\left(-i \epsilon_{n}+\xi_{l}\left(k_{z}\right)\right) \psi_{l, k_{y}}\left(k_{z}, \epsilon_{n}\right),
$$

where $\psi, \bar{\psi}$ are the electron field operators in the energy eigenbasis, $\epsilon_{n}=(2 n+1) \pi k_{B} T$ is the Matsubara frequency, and $\xi_{l}\left(k_{z}\right)=\hbar \omega_{c}(l+1 / 2)-2 t_{\perp} \cos \left(k_{z} a_{\perp}\right)-\mu$ is the singleparticle energy measured from the Fermi level. The discrete eigenstate index $s$ of the bilayer is replaced by the continuous quasimomentum $k_{z} \in\left(-\pi / a_{\perp}, \pi / a_{\perp}\right]$ describing dispersion along the $c$ axis, and we use the notation $\int_{k_{z}}=\int_{-\pi / a_{\perp}}^{\pi / a_{\perp}} d k_{z} / 2 \pi$, where $a_{\perp}$ is the interlayer spacing. With our choice of Landau gauge, $k_{y}$ indexes the degenerate states in each Landau level with total number $\sum_{k_{y}}=A n_{\Phi}$, with $A$ the sample area. The frequencies determined by the two extremal crosssectional areas of the Fermi surface along $k_{z}$ are $f_{ \pm}=m^{*}(\mu \pm$ $\left.2 t_{\perp}\right) / e \hbar$ [34]. As before we consider spinless electrons, as this already shows the new effect.

For simplicity, in considering the interlayer interaction between local charge density fluctuations we approximate the (screened) Coulomb potential as only acting between pairs of nearest points on neighboring layers, $V_{i, i^{\prime}}\left(\mathbf{r}-\mathbf{r}^{\prime}\right)=$ $\delta_{i^{\prime}, i+1} e^{2} / 4 \pi \varepsilon\left|\mathbf{r}-\mathbf{r}^{\prime}+a_{\perp} \hat{\mathbf{z}}\right| \approx V \lambda^{2} \delta_{i^{\prime}, i+1} \delta\left(\mathbf{r}-\mathbf{r}^{\prime}\right)$, where $\varepsilon$ is the permittivity and $\lambda^{2}$ is the area of the "patch" on each layer that participates in the interaction. The interaction term in the action is

$$
S_{\mathrm{int}} \approx V \lambda^{2} \int_{0}^{\beta} d \tau \int d \mathbf{r} \sum_{i} \bar{c}_{i}(x) \bar{c}_{i+1}(x) c_{i+1}(x) c_{i}(x),
$$


where $x=(\tau, \mathbf{r})$. We also acquire a term $S_{I}=$ $\beta A L_{z} V \lambda^{2} n_{I}\left(n_{I} / a_{\perp}-2 n\right)$, where $L_{z}$ is the extent of the system in $z$, which depends only on the total electron density $n$ and accounts for the interaction between electrons and lattice ions. Note that here $n$ represents a number per unit 3D volume, whereas $n_{I}$ is a number per unit $2 \mathrm{D}$ area in plane.

To analyze the interaction effects we proceed as follows. The interlayer interaction can be included to first order with the Hartree-Fock self-energy $\Sigma$, inserted into the full electron Green's function $G=\left(G_{0}^{-1}-\Sigma\right)^{-1}$, with $G_{0}=\left(i \epsilon_{n}-\xi\right)^{-1}$ the free-electron Green's function. It is important for our analysis to keep both the constant, zero-field part $\Sigma_{0}$ and oscillatory part $\widetilde{\Sigma}(B)$ of the self-energy; many previous studies of the dHvA effect including interactions have used the general relation $\widetilde{\Sigma} \ll \Sigma_{0}$ to discard $\widetilde{\Sigma}$ entirely [21-23], though it has been appreciated that this term can affect the properties of dHvA oscillations in 2D metals [35]. We find that this oscillatory term is responsible for the leading contribution to difference-frequency oscillations in the layered model we examine.

From the Green's function $G$ we calculate the grand potential $\Omega$ via standard field theoretic methods, expanding up to first order in the interaction constant $V$. We then obtain the free energy $F$ as the Legendre transform of the grand potential, $F(B, n)=\Omega[B, \mu(B, n)]+n \mu(B, n)$, where $n$ is the fixed electron density, and $\mu(B, n)$ is the oscillatory chemical potential needed to fix the electron density. To linear order in $V$, it is sufficient to fix the density using just the noninteracting part of the theory, i.e., defining $\mu(B, n)$ through $n=-\partial \Omega /\left.\partial \mu\right|_{V=0}$; corrections to $\mu$ that depend on $V$ only lead to terms in $F$ that are of order $V^{2}$ and higher [36]. The result of this procedure - the free energy $F(B, n)$-is the relevant thermodynamic potential for a system with fixed electron density, including all first-order interlayer interaction effects.

We calculate the Hartree-Fock self-energy in the approximation that the interaction is independent of the Landau-level index,

$$
\begin{gathered}
\Sigma\left(k_{z}, B\right)=V \lambda^{2} a_{\perp}\left(n(B)-\chi(B) \cos \left(k_{z} a_{\perp}\right)\right), \\
n(B)=\int_{k_{z}} n\left(k_{z}, B\right), \quad \chi(B)=\int_{k_{z}} \cos \left(k_{z} a_{\perp}\right) n\left(k_{z}, B\right) .
\end{gathered}
$$

This gives the exact form of $\Omega$ up to first order in $V$. Here, we define the 3D number density of occupied states at momentum $k_{z}, n\left(k_{z}, B\right)=n_{\Phi} \int d \epsilon \sum_{l} n_{F}(\epsilon) \mathcal{A}\left(\epsilon-\xi_{l}\left(k_{z}\right)\right)$, where $\mathcal{A}$ is the spectral density. With the 3D electron density fixed to $n(B)=$ $n=n_{I} / a_{\perp}$ we determine the oscillatory chemical potential $\mu(B, n)[36]$, then obtain the free energy $F_{3 \mathrm{D}}=F_{3 \mathrm{D}}^{(0)}+F_{3 \mathrm{D}}^{(1)}$ with

$$
F_{3 \mathrm{D}}^{(0)}=n_{\Phi} \int_{k_{z}} \int d \epsilon \epsilon n_{F}(\epsilon) \sum_{l} \mathcal{A}\left(\epsilon-\xi_{l}\left(k_{z}\right)\right)+n \mu(B, n)
$$

$$
F_{3 \mathrm{D}}^{(1)}=-V \lambda^{2} a_{\perp} \chi(B, \mu(B, n))^{2} .
$$

As in the $2 \mathrm{D}$ case, the interaction part $F_{3 \mathrm{D}}^{(1)}$ is given solely by the Fock energy.

We analytically evaluate the oscillatory part of the free energy assuming the hierarchy of energy scales $\hbar \omega_{c} \ll$ $2 t_{\perp} \ll \mu$. Equivalently, this sets a hierarchy of $2 \mathrm{D}$ densities
$n_{\Phi} \ll n_{\perp} \ll n_{I}$, where we define $n_{\perp} \equiv t_{\perp} m^{*} / \pi \hbar^{2}$. The dominant contributions to oscillations at $f_{ \pm}$and $\delta f$ are found to be [36]

$$
\begin{aligned}
\widetilde{F}_{3 \mathrm{D}}^{(0)}= & \frac{\hbar \omega_{c} n_{\Phi}^{2}}{8 \pi^{4} a_{\perp} n_{\perp}} R_{D, 1}^{2} R_{T, 1}^{2} \sin \left(\frac{2 \pi \delta f}{B}\right) \\
& -\frac{\hbar \omega_{c} n_{\Phi}}{4 \pi^{3} a_{\perp}} \sqrt{\frac{n_{\Phi}}{n_{\perp}}} R_{D, 1} R_{T, 1} \sum_{\alpha= \pm} \cos \left(\frac{2 \pi f_{\alpha}}{B}-\frac{\alpha \pi}{4}\right)
\end{aligned}
$$

from the kinetic part, and

$$
\begin{aligned}
\widetilde{F}_{3 \mathrm{D}}^{(1)}= & V \lambda^{2} \frac{n_{\Phi}^{2}}{2 \pi^{3} a_{\perp}} R_{D, 1}^{2} R_{T, 1}^{2} \cos \left(\frac{2 \pi \delta f}{B}\right) \\
& +V \lambda^{2} \frac{n_{\Phi} n_{\perp}}{2 \pi^{2} a_{\perp}} \sqrt{\frac{n_{\Phi}}{n_{\perp}}} R_{D, 1} R_{T, 1} \sum_{\alpha= \pm} \alpha \sin \left(\frac{2 \pi f_{\alpha}}{B}-\frac{\alpha \pi}{4}\right)
\end{aligned}
$$

from the interacting part, where

$$
R_{D, p}=\exp \left[-\frac{\pi p}{\omega_{c} \tau_{\mathrm{qp}}}\right], \quad R_{T, p}=\frac{2 \pi^{2} p \frac{k_{B} T}{\hbar \omega_{c}}}{\sinh \left(2 \pi^{2} p \frac{k_{B} T}{\hbar \omega_{c}}\right)},
$$

are the Dingle factor and LK temperature factor, accounting for finite quasiparticle lifetime $\tau_{\mathrm{qp}}$ and nonzero temperature $T$, respectively. Note that in both Eqs. (11) and (12) the $\delta f$ terms are found to depend on the square of these factors, while the $f_{ \pm}$terms only depend on a single power of each.

We verify our analytic calculations by analyzing the clean, $T \rightarrow 0$ limit of the system numerically. The chemical potential to fix $n$ is evaluated numerically, the full $F_{3 \mathrm{D}}^{(0)}$ and $F_{3 \mathrm{D}}^{(1)}$ are evaluated on a set of points evenly spaced in $1 / B$, a polynomial background is subtracted off, and the spectral content of the resulting oscillatory data is analyzed via a discrete Fourier transform. The resulting Fourier spectra are presented in fig. 1 . The amplitudes at $f_{ \pm}$and $\delta f$ given by this analysis are found to closely match the $\tau_{\mathrm{qp}} \rightarrow \infty, T \rightarrow 0$ limit of the analytic results, Eqs. (11) and (12).

Discussion. In the above analysis we have determined the oscillations of the free energy of the 3D system. The oscillatory part of the magnetization can be obtained as $\widetilde{M}=$ $-\partial \widetilde{F}_{3 \mathrm{D}} / \partial B$, and naturally separates into interacting and noninteracting parts in the same way as $F_{3 \mathrm{D}}, \widetilde{M}=\widetilde{M}^{(0)}+\widetilde{M}^{(1)}$. The largest contributions arise from the derivative acting on the oscillating factors themselves, not the preceding amplitudes, as long as $k_{B} T, \hbar / \tau_{\mathrm{qp}} \ll t_{\perp}$. Thus, the oscillation amplitudes of $\widetilde{M}$ can be acquired from those of $\widetilde{F}_{3 \mathrm{D}}$ in Eqs. (11) and (12) by simply multiplying each term with a factor of $2 \pi f / B^{2}$, where $f$ is the oscillation frequency of that term.

To compare the predictions of this theory with the experimental observations on $\mathrm{PtCoO}_{2}$, we use the parameters reported in Ref. [20] (see Ref. [37]). The investigation therein of short-range interactions in this material suggests an on-site Hubbard-like repulsion with $U \simeq 6 \mathrm{eV}$. We take this to suggest short-range Coulomb interaction energies in 

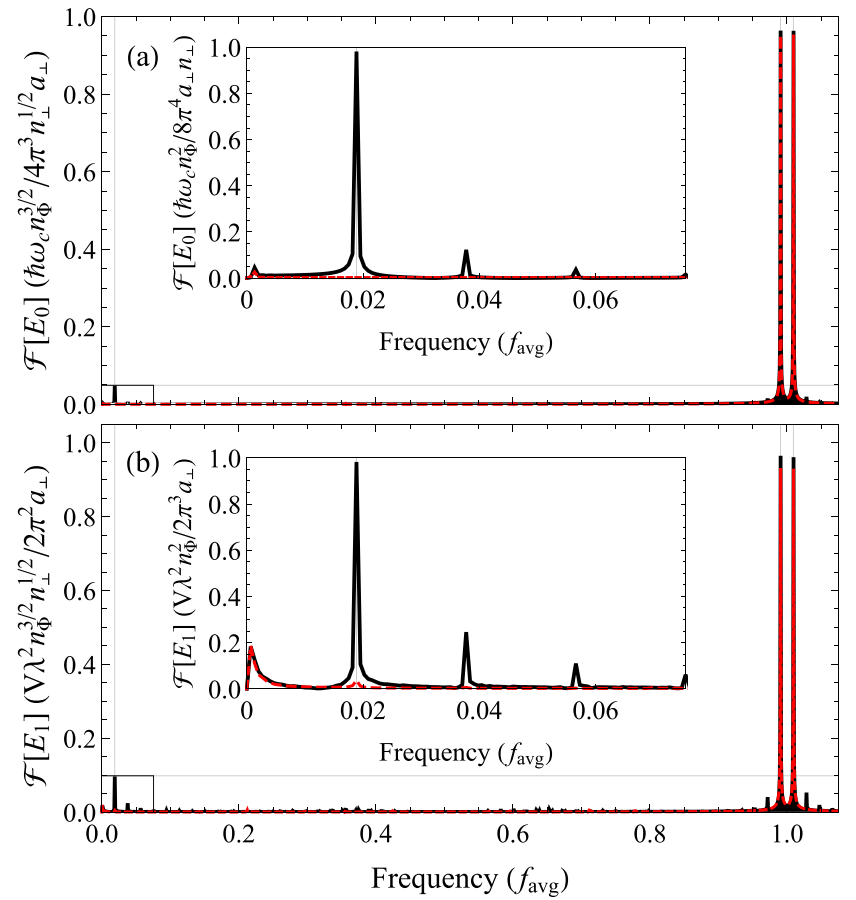

FIG. 1. The Fourier spectra of the (a) kinetic energy $F_{3 \mathrm{D}}^{(0)}$ and (b) Fock interaction energy $F_{3 \mathrm{D}}^{(1)}$ for the 3D multilayer system. The red dashed lines show the same spectra for the case of a fixed chemical potential [36]. Frequencies are given in units of $f_{\text {avg }}=$ $\left(f_{+}+f_{-}\right) / 2=m^{*} \mu / e \hbar$. We use $\delta f / f_{\text {avg }}=4 t_{\perp} / \mu=0.0189$, consistent with the parameters of $\mathrm{PtCoO}_{2}$ [20]. Vertical and horizontal guidelines mark the location and size of the largest differencefrequency peak in each panel. The insets of each panel expand the boxed sections of the main figures. The vertical scale of each panel is set by the appropriate amplitude in Eq. (11) or (12).

general, including $V$, are on the scale of $\mathrm{eV}$, and use $\lambda^{2}$ on the scale of the in-plane area of the $\mathrm{PtCoO}_{2}$ unit cell. With these parameters, we find that the difference-frequency amplitude of $\widetilde{M}^{(0)}$ is less than that of $\widetilde{M}^{(1)}$ for all fields $B<4 \pi V \lambda^{2} n_{\perp} m^{*} / \hbar e \sim 600 \mathrm{~T}$. The experimentally relevant low-field regime is far below this threshold, so within our theory dHvA oscillations at $\delta f$ are dominated by the interaction contribution $\widetilde{M}^{(1)}$. This amplitude is given by $\left|\widetilde{M}^{(1)}\right|_{\delta f}=$ $2 V \lambda^{2} \delta f R_{D, 1}^{2} R_{T, 1}^{2} /\left(\pi^{2} \Phi_{0}^{2} a_{\perp}\right)$, where, assuming spin splitting to be small, we now include the effect of spin via an overall factor of 2. Note that this depends on magnetic field strength only through the squares of the Dingle and LK temperature factors, while the corresponding amplitude of the noninteracting term $\widetilde{M}^{(0)}$ depends on an additional factor of $B$ itself. This form of $\left|\widetilde{M}^{(1)}\right|_{\delta f}$ qualitatively matches the experimental results of the field dependence of the low-frequency oscillations in Ref. [20], and its magnitude is of the same order of magnitude as the measured effect [38]. Future measurements, focusing on the quantitative size of this low-frequency component, have the potential to allow for a better understanding of the strength and nature of interlayer interactions.

The careful reader may note that the inequality $\delta f \ll f_{ \pm}$, inherited from $t_{\perp} \ll \mu$, suggests that the dHvA oscillations our theory predicts at $\delta f$ should always be much smaller than those at $f_{ \pm}$, contrasting with Ref. [20] where they are observed to be comparably sized. But since our theory is in decent agreement with experiment regarding the $\delta f$ oscillation amplitude, the discrepancy lies in an overestimate of the size of the $f_{ \pm}$oscillations, for which our theory coincides with standard LK theory. Indeed this same anomaly was noted in the experimental findings of Ref. [20], where the size of the $f_{ \pm}$ oscillations was observed to be much smaller than that anticipated from LK theory using the Dingle factor computed from the mean-free path. This apparent problem can be resolved by considering long-range disorder, producing a slowly varying spatial inhomogeneity of the average $\mu$ (or the fixed local density) and small-angle scattering. Long-wavelength spatial inhomogeneity is known to significantly suppress QO amplitudes through phase smearing [21,39] without a significant impact on transport, suggesting that this observation may be related to long-range disorder in the system.

Importantly, the difference-frequency oscillations are insensitive to the phase smearing effect, being entirely independent of $\mu$. Therefore, long-range disorder strongly suppresses only the high-frequency oscillations, and allows oscillations at $\delta f$ and $f_{ \pm}$to be of a comparable scale. Thus our theory naturally recovers the observation of Ref. [20], that the Dingle suppression of the high-frequency oscillations is much larger than that expected from the transport lifetime, while the Dingle suppression of the difference-frequency oscillation is much closer to that based just on the transport lifetime. This starkly different dependence on sample inhomogeneity suggests that some measure of long-range disorder may be obtained by comparing Dingle measurements of difference and main frequency oscillations.

We also compare the size of this Coulomb interaction effect to that generated by magnetic interactions [21]. The magnetic interaction effect at $\delta f$ is determined by the product of the oscillation amplitudes of the magnetization at $f_{ \pm}$, which we can determine from Eq. (11). Since long-range disorder strongly suppresses these oscillations, as noted above, this will likewise be strongly suppressed. Using the same parameters as above and the Dingle temperatures identified for lowand high-frequency oscillations in Ref. [20], we find that the magnetic interaction contribution to oscillations at $\delta f$ is much smaller than the Coulomb interaction effect we identify, being $\sim 10^{-7}-10^{-3}$ times smaller for $B=7.5-35 \mathrm{~T}$ [36].

In summary, by considering oscillations of the selfenergy that are neglected in standard theories [21-23], we have shown that Coulomb interactions can give rise to sizable low-frequency dHvA oscillations in high-mobility layered materials. These difference-frequency oscillations are highly sensitive to short-range disorder, their amplitude being suppressed by the square of the Dingle factor, but are insensitive to long-range disorder. They can therefore become a dominant feature of oscillations in very high-mobility materials such as the delafossites. Our theory shows that the size of the difference-frequency oscillation is a measure of the strength and form of the interactions beyond the on-site Hubbard $U$. Although we have focused on simple layered metals, our results suggest that dHvA studies may be of use to probe properties beyond just Fermisurface geometry in more general bilayer and multilayer systems. 
Acknowledgment. We thank Johannes Knolle for helpful discussions, and thank also Elena Hassinger and Andrew Mackenzie for helpful discussions, for sharing experimental data, and for comments on an earlier draft. This work is supported by EPSRC Grant No. EP/P034616/1 and by a Simons Investigator Award.
[1] W. J. de Haas and P. M. van Alphen, The dependence of the susceptibility of diamagnetic metals upon the field, Proc. Neth. R. Acad. Sci. 33, 1106 (1930).

[2] I. Lifshitz and A. Kosevich, Theory of magnetic susceptibility in metals at low temperature, Sov. Phys. - JETP 2, 636 (1956).

[3] A. C. Potter, I. Kimchi, and A. Vishwanath, Quantum oscillations from surface Fermi arcs in Weyl and Dirac semimetals, Nat. Commun. 5, 5161 (2014).

[4] F. Arnold, M. Naumann, S.-C. Wu, Y. Sun, M. Schmidt, H. Borrmann, C. Felser, B. Yan, and E. Hassinger, Chiral Weyl Pockets and Fermi Surface Topology of the Weyl Semimetal TaAs, Phys. Rev. Lett. 117, 146401 (2016).

[5] A. Alexandradinata and L. Glazman, Geometric Phase and Orbital Moment in Quantization Rules for Magnetic Breakdown, Phys. Rev. Lett. 119, 256601 (2017).

[6] A. Alexandradinata and L. Glazman, Semiclassical theory of landau levels and magnetic breakdown in topological metals, Phys. Rev. B 97, 144422 (2018).

[7] N. L. Nair, M.-E. Boulanger, F. Laliberté, S. Griffin, S. Channa, A. Legros, W. Tabis, C. Proust, J. Neaton, L. Taillefer, and J. G. Analytis, Signatures of possible surface states in TaAs, Phys. Rev. B 102, 075402 (2020).

[8] T. Devakul, Y. H. Kwan, S. L. Sondhi, and S. A. Parameswaran, Quantum Oscillations in the Zeroth Landau Level: Serpentine Landau Fan and the Chiral Anomaly, Phys. Rev. Lett. 127, 116602 (2021).

[9] Y. Zhang, A. V. Maharaj, and S. Kivelson, Disruption of quantum oscillations by an incommensurate charge density wave, Phys. Rev. B 91, 085105 (2015).

[10] S. Spurrier and N. R. Cooper, Theory of quantum oscillations in quasicrystals: Quantizing spiral Fermi surfaces, Phys. Rev. B 100, 081405(R) (2019).

[11] B. S. Tan, Y.-T. Hsu, B. Zeng, M. C. Hatnean, N. Harrison, Z. Zhu, M. Hartstein, M. Kiourlappou, A. Srivastava, M. D. Johannes, T. P. Murphy, J.-H. Park, L. Balicas, G. G. Lonzarich, G. Balakrishnan, and S. E. Sebastian, Unconventional Fermi surface in an insulating state, Science 349, 287 (2015).

[12] J. Knolle and N. R. Cooper, Quantum Oscillations without a Fermi Surface and the Anomalous de Haas-van Alphen Effect, Phys. Rev. Lett. 115, 146401 (2015).

[13] G. Baskaran, Majorana Fermi sea in Insulating $\mathrm{SmB}_{6}$ : A proposal and a theory of quantum oscillations in Kondo insulators, arXiv:1507.03477.

[14] O. Erten, P. Ghaemi, and P. Coleman, Kondo Breakdown and Quantum Oscillations in $\mathrm{SmB}_{6}$, Phys. Rev. Lett. 116, 046403 (2016).

[15] L. Zhang, X.-Y. Song, and F. Wang, Quantum Oscillation in Narrow-Gap Topological Insulators, Phys. Rev. Lett. 116, 046404 (2016).

[16] J. Knolle and N. R. Cooper, Anomalous de Haas-van Alphen Effect in InAs/GaSb Quantum Wells, Phys. Rev. Lett. 118, 176801 (2017).
[17] I. Sodemann, D. Chowdhury, and T. Senthil, Quantum oscillations in insulators with neutral Fermi surfaces, Phys. Rev. B 97, 045152 (2018).

[18] M. Hartstein, W. H. Toews, Y.-T. Hsu, B. Zeng, X. Chen, M. C. Hatnean, Q. R. Zhang, S. Nakamura, A. S. Padgett, G. RodwayGant, J. Berk, M. K. Kingston, G. H. Zhang, M. K. Chan, S. Yamashita, T. Sakakibara, Y. Takano, J.-H. Park, L. Balicas, N. Harrison et al., Fermi surface in the absence of a Fermi liquid in the Kondo insulator $\mathrm{SmB}_{6}$, Nat. Phys. 14, 166 (2018).

[19] C. W. Hicks, A. S. Gibbs, A. P. Mackenzie, H. Takatsu, Y. Maeno, and E. A. Yelland, Quantum Oscillations and High Carrier Mobility in the Delafossite $\mathrm{PdCoO}_{2}$, Phys. Rev. Lett. 109, 116401 (2012).

[20] F. Arnold, M. Naumann, H. Rosner, N. Kikugawa, D. Graf, L. Balicas, T. Terashima, S. Uji, H. Takatsu, S. Khim, A. P. Mackenzie, and E. Hassinger, Fermi surface of $\mathrm{PtCoO}_{2}$ from quantum oscillations and electronic structure calculations, Phys. Rev. B 101, 195101 (2020).

[21] D. Shoenberg, Magnetic Oscillations in Metals, Cambridge Monographs on Physics (Cambridge University Press, Cambridge, UK, 1984).

[22] J. M. Luttinger, Theory of the de Haas-van Alphen Effect for a System of Interacting Fermions, Phys. Rev. 121, 1251 (1961).

[23] A. Wasserman and M. Springford, The influence of many-body interactions on the de Haas-van Alphen effect, Adv. Phys. 45, 471 (1996).

[24] G. W. Crabtree, Demagnetizing fields in the de Haas-van Alphen effect, Phys. Rev. B 16, 1117 (1977).

[25] T. Champel and V. P. Mineev, de Haas-van Alphen effect in two- and quasi-two-dimensional metals and superconductors, Philos. Mag. B 81, 55 (2001).

[26] P. Grigoriev, The influence of the chemical potential oscillations on the de Haas-van Alphen effect in quasi-two-dimensional compounds, J. Exp. Theor. Phys. 92, 1090 (2001).

[27] P. D. Grigoriev, Theory of the Shubnikov-de Haas effect in quasi-two-dimensional metals, Phys. Rev. B 67, 144401 (2003).

[28] A. S. Alexandrov and A. M. Bratkovsky, de Haas-van Alphen Effect in Canonical and Grand Canonical Multiband Fermi Liquid, Phys. Rev. Lett. 76, 1308 (1996).

[29] M. Nakano, Unexpected de Haas-van Alphen oscillation in 2D multiband systems due to chemical potential oscillation and its relevance to magnetic breakdown systems, J. Phys. Soc. Jpn. 66, 19 (1997)

[30] J.-Y. Fortin and T. Ziman, Frequency Mixing of Magnetic Oscillations: Beyond Falicov-Stachowiak Theory, Phys. Rev. Lett. 80, 3117 (1998).

[31] T. Champel, Origin of combination frequencies in quantum magnetization oscillations of two-dimensional multiband metals, Phys. Rev. B 65, 153403 (2002).

[32] K. Kishigi and Y. Hasegawa, de Haas-van Alphen effect in twodimensional and quasi-two-dimensional systems, Phys. Rev. B 65, 205405 (2002). 
[33] The numerical values of the prefactors of these two terms can vary, depending on the spatial form of the interelectron interactions. The form in (2) arises for the model interactions chosen in (6), of short-range interlayer interactions.

[34] We extract numerical values for $\mu$ and $t_{\perp}$ by fitting the frequencies reported in Ref. [20] to this form.

[35] K. Miyake and C. Varma, Many body effect on oscillatory properties of two-dimensional metals in a magnetic field, Solid State Commun. 85, 335 (1993).
[36] See Supplemental Material at [http://link.aps.org/supplemental/ 10.1103/PhysRevResearch.3.L042009] for calculations of free energies and comparison to magnetic interactions.

[37] Explicitly, we use $m^{*}=1.18 m_{e}$, with $m_{e}$ the free-electron mass, $\mu=2.963 \mathrm{eV}, t_{\perp}=13.98 \mathrm{meV}$, and $c$-axis lattice constant $a_{\perp}=17.808 \AA$.

[38] E. Hassinger (private communication).

[39] As noted in Ref. [21], phase smearing due to inhomogeneity often produces a stronger suppression than a finite quasiparticle lifetime. 